\title{
Indicadores de competitividad del cultivo del arroz en Colombia y Estados Unidos
}

\author{
Competitive indicators from Colombia and U.S. rice production
}

Jaqueline Chica L. ; Yeimy Carolina Tirado 0.'; Joan Manuel Barreto 0. ${ }^{3}$

\author{
Docente. MSc. Universidad del Tolima. Ibagué, Colombia, jchica@ut.edu.co. \\ Ingeniera Agrónomo. Grupo de Investigación Desarrollo Rural Sostenible. Ibagué, Colombia, yctiradoo@ut.edu.co. \\ Ingeniero Agrónomo. Universidad del Tolima. Ibagué, Colombia, jmbarreto@ut.edu.co.
}

Citar: CHICA, J.; TIRADO, Y.; BARRETO, J. 2016. Indicadores de competitividad del cultivo del arroz en Colombia y Estados Unidos. 33(2): 16-31. doi: http://dx.doi.org/10.22267/rcia.163302.49.

Recibido: Abril 26 de $2016 . \quad$ Aceptado: Septiembre 26 de 2016.

\section{RESUMEN}

En los últimos 20 años, Colombia ha venido profundizando su inserción en el mercado mundial mediante la firma de tratados de libre comercio con diferentes países; a pesar de su vulnerabilidad, el sector agrícola ha sido vinculado en estos acuerdos comerciales. El arroz, es el tercer cultivo en importancia del país y fue incluido como producto de importación en el TLC con Estados Unidos, desde entonces, se ha cuestionado su permanencia en el campo colombiano. Este estudio evaluó la competitividad del sector arrocero colombiano y estadounidense, mediante indicadores de competitividad revelada y cuasi rentas, identificando la incidencia de factores económicos y no económicos, con el objetivo de evaluar el impacto del TLC en la producción arrocera colombiana y su capacidad para sostenerse en el mercado nacional. Los indicadores de competitividad revelada para Colombia, reflejaron valores negativos y cercanos a cero, esto es, niveles de competitividad nulos; mientras para Estados Unidos los niveles fueron cercanos a uno, evidenciando su fortaleza como competidor en el mercado internacional. De igual manera, los indicadores de cuasi rentas evidenciaron mayor competitividad por parte del sector estadounidense. Esto se explica por el uso de una tecnología de producción avanzada y muy eficiente, e instrumentos de política que van desde inversión en infraestructura de posproducción y transporte hasta subvenciones directas a los productores. El TLC con Estados Unidos podría amenazar la seguridad alimentaria nacional de mantenerse la baja competitividad de los arroceros colombianos, pues el aumento de los precios internacionales influye negativamente en la disponibilidad y acceso al arroz.

Palabras clave: Sector arrocero, Tratado de libre comercio, Cuasi- rentas, indicadores de competitividad revelada. 


\begin{abstract}
Colombia has been deepen its integration into the world market through free trade agreements with different countries, for the last twenty years. Despite its vulnerability, the agricultural sector has been linked to these agreements. Rice, is the third most important crop in the country and it was included in the agreement with the United States. Since then, its prevalence as a mayor crop is questioned. The current study evaluated the competitiveness of the Colombian and United States rice sector, through revealed competitiveness and quasi-rents indicators; identifying the influence of economic and non-economic factors, with the aim to evaluate the impact of the Free Trade Agreement in the Colombian rice production and its capability of sustaining by itself in the national market. The Colombian revealed competitiveness indicators show negative and close to zero values, in other words, null levels of competitiveness; in contrast the USA competitiveness levels were close to one, showing its strength as a competitor in the international arena. Similarly, the quasi rent indicator revealed a higher competitiveness of USA's rice sector. This is explained by the use of very efficient and advanced production technology, political instruments involving investment in post-production infrastructure and transportation, and direct subsidies for the producers. The Free Trade Agreement with the USA could threaten the national food security if low competitiveness of the rice producers is maintained, since the increase of the international prices negatively influence rice supply and accessibility.
\end{abstract}

Key words: rice sector, free trade agreement, revealed comparative advantage indicators, quasi-rents indicator.

\section{INTRODUCCIÓN}

La agricultura es una actividad económica fundamental en países en desarrollo como Colombia, toda vez que es la principal fuente de empleo y generación de ingresos para el sector rural (Perfetti et al., 2013). En Colombia, el café, el maíz y el arroz, han sido en su orden los cultivos de mayor importancia productiva en las tres últimas décadas debido al área cultivada, volumen de producción y generación de empleo (AGRONET, 2014); no obstante, su importancia económica es significativamente diferente (Garay et al., 2005). El arroz es considerado el cultivo más importante del mundo, además de ser un producto básico como el maíz, posee la mayor extensión de tierra cultivada y el mayor número de personas dedicadas a su producción. Casi el 50\% de la población mundial, depende del arroz como parte importante de su dieta, el consumo per cápita mundial para 2011, estuvo alrededor de los 65 kilogramos. (Mohanty, 2013). La producción de arroz en Colombia, genera alrededor de 500 mil empleos directos e indirectos, en más de 215 municipios, los cuales dependen en un 90\% de ésta actividad (FEDEARROZ, 2014). Según el III Censo Nacional Arrocero, existen aproximadamente 21.800 productores, en 26.733 UPA (Unidades Productoras de Arroz), distribuidas en las cinco zonas productoras del país (FEDEARR0Z, 2008). El consumo per cápita de arroz en Colombia, es mayor que en Estados Unidos de América (EUA); en el año 2012 se registró en promedio, un consumo de $35,2 \mathrm{Kg} / \mathrm{año}$ y de $8,3 \mathrm{Kg} / \mathrm{año}$, respectivamente, aunque ambos consumos están por debajo del promedio mundial (53,3Kg/año) (FAOSTAT, 2012). Tradicionalmente, Colombia ha satisfecho su demanda interna con producción propia y realiza importaciones ocasionales en pequeños volúmenes; sin embargo, desde 2012, año en que entra en vigencia el TLC con EUA, algunos problemas fitosanitarios, el cambio climático, junto a la posibilidad de importar mayores volúmenes desde el primer año del tratado, ha marcado una tendencia de crecimiento 
de las importaciones y una disminución del área cultivada, por el desestimulo que el tratado ha generado entre los productores.

Esta investigación, parte de la expectativa racional que altos rendimientos por unidad de área y menores precios del arroz de EUA representan una amenaza para los productores colombianos, quienes podrían reconvertirse a otros cultivos y en consecuencia aumentaría la dependencia a las importaciones para suplir el consumo nacional, lo que a futuro podría generar escenarios de inseguridad alimentaria para la población colombiana de menor ingreso, en coyunturas de altos precios internacionales como los de 2005-2008 y 2011- 2012 (Ramírez et al., 2014). Por lo anterior, se evaluó el sector productor de arroz colombiano, frente al estadounidense mediante indicadores de competitividad revelada (ICR) y el indicador de cuasi rentas, midiendo la competitividad de ambos países y su posible evolución en el marco del TLC, con el objetivo de analizar los alcances de dicho tratado en la producción de arroz en Colombia.

La competitividad, es un término utilizado en muchos escenarios, no obstante no existe un total acuerdo sobre su definición conceptual, aunque se coincide en su carácter multidimensional (Balzaraviciene y Pilinkiene, 2012). En cuanto a la semántica del término, esta palabra se asocia a la competencia y a la acción de competir y en tal sentido es utilizada en el discurso común, para hablar indistintamente de competitividad de una firma, un sector económico o un país. Desde la teoría económica clásica, el término ha evolucionado con las teorías del crecimiento y el comercio internacional, reflejando el paso del enfoque de las ventajas comparativas al enfoque de las ventajas competitivas (Garay, 1998; Linares y Gutiérrez, 2002). La discusión conceptual sobre competitividad, involucra la identificación de variables del crecimiento y del desarrollo económico que la explican, como productividad, cambio tecnológico, capital humano, cómo la firma y las naciones pueden hacerse competitivas, hasta propuestas teóricas en las que la competitividad es un factor del bienestar social (Lotero et al., 2005).

Por otra parte, en la medida en que la competitividad se convirtió en un factor obligado de la política pública para el desarrollo, más que su concepto, importa su evaluación y los indicadores que permiten su medición, que será acorde con el nivel de evaluación (firma, sector, región o país) y para ello, se han construido muchos indicadores. A nivel país por ejemplo, el Foro Económico Mundial, publica cada año el Índice de Competitividad Global con base en 114 variables (DNP, 2014) y su metodología, se ha venido utilizando igualmente, para establecer indicadores de competitividad por departamentos en Colombia (Lotero et al., 2009). En éste estudio, la medición se enfoca a un subsector productivo como lo es el arroz. De acuerdo con Rojas y Sepúlveda (1999) la evaluación de la competitividad de un sector productivo, implica la consideración de aspectos micro económicos a nivel de empresas, aspectos de desarrollo de la infraestructura económica de una región (nivel meso) y elementos sociales, relacionados con la cultura de producción y uso de los recursos naturales (nivel meta). Hertford y Espinal (1996) identifican varios tipos de indicadores de competitividad para evaluar un sector productivo (productos, empresas y cadenas productivas), teniendo así, indicadores de tipo holístico, los cuales incluyen diamante de la competitividad nacional, costo de los recursos domésticos y método de las cuasi-rentas, tipo intuitivos, indicadores de competitividad revelada, basados en precios, tasa nominal de protección, tasa de protección efectiva, subsidio equivalente al productor, entre otros; basados en productividad, productividad total o parcial de los factores productivos. La selección de los indicadores de competitividad sectorial utilizados en este trabajo, pretenden medir y comparar la competitividad entre dos países que comparten un tratado de libre comercio, pero fundamentalmente identificar las variables que explican los niveles de 
competitividad alcanzados en cada país, a fin de comprender el alcance que el acuerdo comercial podría tener en el sector productivo estudiado.

\section{MATERIALES Y MÉTODOS}

La evaluación de la competitividad del sector arrocero, se hizo desde un punto de vista agronómico, sin incluir procesos industriales, mediante indicadores de competitividad revelada (ICR) y de las cuasi-rentas, para EUA y Colombia, tratando de identificar factores económicos y no económicos de mayor incidencia en cada caso, basado en información secundaria con validación de algunos expertos en el caso colombiano. Esto permitió identificar las fuertes debilidades que en materia productiva tienen los productores locales.

Se calcularon indicadores de competitividad revelada, que son indicadores indirectos pues no identifican los factores determinantes de la misma; estos indicadores demuestran la capacidad que tiene determinado producto o agrocadena para penetrar o mantenerse en mercados internacionales en un espacio de tiempo, mediante el uso de los valores de la producción, exportación e importación de un producto. Para el caso de éste estudio, se realizó por medio de los valores promedio, entre los años 1990-2010 de las exportaciones, importaciones y producción de arroz total en toneladas para Colombia, Estados Unidos y el mundo, cifras obtenidas a través de FAOSTAT (2015).

Los indicadores de competitividad revelada utilizados en el estudio se listan y describen a continuación, de acuerdo a Espinal et al., (2005). En todos los casos, los indicadores utilizados indican la posición relativa de Colombia y de Estados Unidos en el mercado mundial del arroz, para el período analizado.

$$
\begin{aligned}
& \text { Indicador de Balanza Comercial Relativa } \\
& \qquad \mathbf{B C R}=\left(\left(\mathbf{X}_{\mathrm{ij}}-\mathbf{M}_{\mathrm{ij}}\right) /\left(\mathbf{X}_{\mathrm{ij}}+\mathbf{M}_{\mathrm{ij}}\right)\right.
\end{aligned}
$$

$$
\begin{gathered}
\text { Indicador de transabilidad } \\
\mathrm{T}_{\mathrm{ij}}=\left(\mathbf{X}_{\mathrm{ij}}-\mathbf{M}_{\mathrm{ij}}\right) /\left(\mathbf{Q}_{\mathrm{ij}}+\mathbf{M}_{\mathrm{ij}}-\mathbf{X}_{\mathrm{ij}}\right)
\end{gathered}
$$

Grado de apertura exportadora

$$
\mathrm{GAE}=\mathrm{X}_{\mathrm{ij}} /\left(\mathbf{Q}_{\mathrm{ij}}+\mathrm{M}_{\mathrm{ij}}-\mathrm{X}_{\mathrm{ij}}\right)
$$

Grado de penetración de las importaciones $\mathbf{G P I}=$

$$
M_{i j} /\left(Q_{i j}+M_{i j}-X_{i j}\right)
$$

Indicador de especialización internacional

$$
E I=\left(X_{i j}-M_{i j}\right) /\left(X_{i m}\right)
$$

Donde: $X_{\mathrm{ij}}$ : exportaciones del producto i en el país $\mathrm{j}$; $\mathrm{X}_{\mathrm{im}}$ : exportaciones del bien i realizadas por el mundo; $\mathrm{M}_{\mathrm{iij}}$ : importaciones del producto i en el país j; $Q_{\mathrm{ij}}$; producción del producto i en el país j.

Indicador de Balanza Comercial Relativa (BCR). Este indicador se utiliza para medir la relación entre la balanza comercial neta y el flujo total de exportaciones e importaciones con respecto al mismo producto, permitiendo establecer el grado de ventaja o desventaja comparativa existente. Se tiene ventaja comparativa con valores positivos y no se tiene con valores negativos. Cuando el valor del índice es de $100 \%$ se deduce que la unidad productiva está totalmente orientada a la exportación; cuando el valor es de $-100 \%$ se trata de un producto importable (Alvarado et al. 2009).

Indicador de transabilidad (T). Mide la relación entre la balanza comercial neta y el consumo aparente de un producto en un país. Cuando el indicador es mayor que cero, se considera a la unidad productiva como exportadora, en vista de que existe un exceso de oferta, por lo tanto, el producto es competitivo en el mercado interno. Pero, si el resultado es menor que cero, se trata de un producto importable y se infiere que la unidad productiva no es competitiva en el mercado interno, ya que existe un exceso de demanda (Alvarado et al., 2009). 
Grado de apertura exportadora (GAE). Indica la participación de las exportaciones de un producto sobre el consumo aparente, refiriéndose así al grado de penetración en un mercado en particular. Espinal et al. (2005) señalan que cuando en un periodo de varios años, el indicador se acerca a cero, la competitividad de un país con respecto al mundo está disminuyendo, pues no existe capacidad para sobrepasar el nivel de producción que necesita el país para satisfacer su demanda interna.

Grado de penetración de las importaciones (GPI). Muestra la relación entre las importaciones de un producto y su consumo nacional aparente. El análisis de este indicador se asemeja al anterior, simplemente se habla de un sector suficientemente competitivo cuando los valores se acercan a cero, en ese sentido pueden dedicar gran parte de su producción nacional para la exportación (Espinal et al., 2005).

Indicador de especialización internacional (EI). Mide la participación del saldo existente en la balanza comercial de un producto en el total de las exportaciones mundiales, por lo tanto se puede analizar la aptitud exportadora de cada país y su capacidad para construir ventajas competitivas que perduren en el tiempo. Cuando el nivel para un país es del $100 \%$, se dice que tiene una alta especialización internacional en el producto, y en efecto el país es el único exportador mundial, pero si el valor es negativo, se afirma que no hay ningún grado de especialización por parte del país, el cual presenta dificultades competitivas (Alvarado et al.2009).

También se calculó el indicador de modo de inserción al mercado internacional-IMI oindicador de Franjzilver, medido por Posicionamiento (IMI P): Tasa anual de crecimiento de las exportaciones del producto i del país j. Eficiencia (IMI E): Tasa anual de crecimiento de la participación porcentual de las exportaciones de un país j, en el total de las exportaciones mundiales del producto $\mathrm{i}$.
Estos indicadores reflejan, que un país es suficientemente competitivo en el bien analizado, cuando es un exportador neto y por el contrario cuando se trata de un bien principalmente importado, se dice que en dicho sector no se tiene competitividad (Schwartz et al., 2007), el indicador IMI señala un modo de inserción óptimo cuando IMI P e IMI E son positivos, un modo de inserción en retirada cuando IMI P e IMI E son negativos, un modo de inserción de oportunidades perdidas cuando IMI $\mathbf{P}$ es positivo e IMI $\mathbf{E}$ es negativo y un modo de inserción de vulnerabilidad cuando IMI $\mathbf{P}$ es negativo e IMI $\mathbf{E}$ es positivo.

En segunda instancia, se calculó el indicador de las cuasi-rentas, el cual se deriva del concepto de Stigler (Gutiérrez, 1999), aquí la cuasi-renta es una variable proxy de la competitividad. El indicador aplicado a la producción agrícola, establece que la cuasi-renta de los factores productivos por hectárea se expresa como: $\mathbf{C}=\mathbf{P}^{*} \mathbf{R}^{*} \mathbf{M}$, donde $\mathrm{C}$ = Cuasi-rentas (dólares/ hectárea), $\mathrm{P}=$ Precio al productor (dólares/tonelada), $\mathrm{R}=$ Rendimientos (t/ha), M = Margen de contribución.

El margen de contribución corresponde a la participación porcentual de las ganancias netas en los ingresos totales, y resulta de restarle al precio al productor, los costos variables promedios y dividirlos entre el precio. Los valores de precio al productor y costos de producción, fueron llevados a precios constantes del año 2000 , las cuasi rentas se calcularon para el promedio de años entre 1991 2011. Éste método, evidencia eficiencia técnica del productor a través de los rendimientos y eficiencia económica por medio del margen de contribución, reflejándose también capacidad administrativa (Gutiérrez, 1999).

Tanto en Colombia como en EUA, la producción arrocera se divide en diferentes zonas edafoclimáticas, las cuales determinan la forma de producción y tecnología empleada. La producción arrocera en Colombia se da en más de 200 municipios, agrupados en cinco zonas productoras 
así: Zona Centro, áreas planas y cálidas de los valles de los ríos Magdalena y Cauca correspondientes a los departamentos de Tolima, Huila, Cundinamarca, Caldas, Boyacá, Cauca, Valle del Cauca y Caquetá; Zona Llanos Orientales, incluye Meta, Casanare y Arauca, y los municipios del piedemonte llanero de Cundinamarca; Zona Bajo Cauca, incluye Antioquia, Bolívar, Córdoba, y Sucre, además municipios arroceros del Chocó y región del Urabá; Zona Costa Norte, incluye Cesar, Atlántico, Magdalena, y Guajira; Zona Santanderes, comprende Santander y Norte de Santander (FEDEARROZ, 2008).

En EUA, la producción de arroz se concentra en Arkansas, California, Louisiana, Mississippi, Missouri y Texas, estados agrupados en cuatro regiones de acuerdo con la clasificación del suelo y otras características distribuidas así: Arkansas Non-Delta, comprende condados al norte de Arkansas y el bootheel de Missouri y la Gran Pradera de Arkansas; Región Delta del Río Mississippi compuesta por condados sureños de Arkansas adyacentes del Río Mississippi y norte de Louisiana; Región Costa del Golfo, incluye sur de Louisiana y los condados de la Llanura Costera del sur de Texas. Las tres regiones abarcan el 80\% del área y producción arrocera en EUA; Región California, estado donde se cultiva exclusivamente arroz de alta a media calidad, dependiendo de las condiciones del mercado se destina al comercio interno o al exportación (Baldwin et al., 2011).

Las cuasi rentas, fueron calculadas para Colombia a nivel nacional y para la zona centro, la cual presenta los mayores rendimientos. En el caso de Estados Unidos, la evaluación se realizó sólo a nivel nacional, debido a la poca diferencia de las variables evaluadas entre las diferentes zonas productoras. Las cuasi rentas se calcularon con y sin los subsidios directos que reciben los productores en Estados Unidos, con el fin de poder mostrar la gran influencia que tienen estos sobre los ingresos de los agricultores.

Para el cálculo de las cuasi rentas en Colombia, la información de precios, rendimientos y costos de producción, provienen de las estadísticas históricas de FEDEARROZ, disponibles en su página web. Para Estados Unidos, los rendimientos y costos de producción se tomaron de las estadísticas del departamento de Agricultura, mientras que los precios se tomaron de FEDEARROZ, teniendo en cuenta que el productor estadounidense vende arroz paddy seco. En cuanto a los subsidios, los tipos, las condiciones y los porcentajes, están establecidos por la ley agrícola de 2008 y los datos para el periodo de análisis se obtuvieron de la página web Environmental Working Group (EWG, 2015).

\section{RESULTADOS Y DISCUSIÓN}

\section{Indicadores de Competitividad Revelada - ICR.} Todos los ICR (Tabla 1) fueron en su conjunto muy bajos o negativos para Colombia, demostrando, que dadas las condiciones actuales de producción del arroz, el país no es competitivo en el mercado internacional. Mientras que los ICR para EUA reflejan un alto nivel competitivo porque su modelo de negocio se ha enfocado a las exportaciones.

Tabla 1. Indicadores de competitividad revelada Colombia y EUA promedio 1990 - 2010.

\begin{tabular}{lccccccc}
\hline Indicador & IT & BCR & GAE & GPI & EI & IMI P & IMI E \\
\hline Colombia & $-4,02$ & $-79,5$ & 0,4 & 4,41 & $-0,35$ & $-32,41$ & $-22,39$ \\
\hline EUA & 41,63 & 77,71 & 47,55 & 5,92 & 11,14 & 3,45 & $-1,48$ \\
\hline
\end{tabular}

Fuente: Cálculos de los autores basados en datos de Faostat s.f. (FAOSTAT, 2015) 
Al analizar el indicador de transabilidad para Colombia en el periodo evaluado, se observó un exceso de demanda de arroz del 4\% lo cual indica que es necesario importar el producto. En el caso de Estados Unidos, el valor medio para el mismo periodo fue de $41,6 \%$, representando esto un exceso de oferta de arroz, lo cual indica que el modelo de negocio de este país es producir arroz para la exportación, sin ser uno de los principales productores.

Balanza comercial relativa. A excepción de 1990 y 1991, Colombia ha presentado una balanza comercial deficitaria, debido a las mínimas exportaciones que se han presentado, pero sobre todo a un aumento progresivo de las importaciones. Este indicador reveló la baja competitividad del sector arrocero colombiano en mercados internacionales y la alta capacidad exportadora de Estados Unidos, el cual no alcanza valores de $100 \%$ debido a que también importa considerables volúmenes de éste producto.

Grado de apertura exportadora. Debido a que éste indicador mide la participación únicamente de las exportaciones en el consumo aparente, los valores obtenidos para Colombia se sitúan en 0 en vista de que desde 1991 las exportaciones se tornaron insignificantes. Espinal et al., (2005), señalan al respecto que si en un periodo de varios años dicho indicador se aproxima a cero, se refleja que la competitividad de un país está decreciendo en comparación con los otros países. Por su parte, los niveles de Estados Unidos reflejan que existe un alto nivel de competitividad.

Grado de penetración de importaciones. Este indicador respalda los valores obtenidos en el indicador de transabilidad, evidenciando que para el caso de Colombia la participación de las importaciones en el consumo aparente fue en promedio para el periodo de 1990-2010, 4.41\%, mientras para Estados Unidos fue 6\%. Esto significa que la producción nacional de ambos países prácticamente satisface la demanda interna demandando un pequeño volumen de arroz en el mercado internacional.

Indicador de especialización internacional. Éste indicador tiene en cuenta la participación del saldo de la balanza comercial de un país, con respecto a las exportaciones mundiales. En ese sentido, al no existir un saldo del producto en Colombia, el indicador define el país como importador neto. No obstante se evidencia que para Estados Unidos los niveles no han sobrepasado el 20\%, lo cual lo puede definir como de baja especialización en la comercialización internacional del arroz, es decir, pese a ser un buen productor de arroz, con una importante participación en el mercado internacional, otros países como Tailandia son mejores competidores.

El indicador de modo de inserción que relaciona posicionamiento y eficiencia, muestra a Colombia, en un escenario de retirada, aunque el país nunca ha sido un fuerte exportador, mientras EUA se encuentra en un escenario de oportunidades perdidas porque si bien sus exportaciones crecieron, otros países han ganado terreno en el mercado mundial del arroz, de allí que busquen nuevos mercados que le permitan explotar sus ventajas competitivas a través de TLC. Sin embargo, los ICR no son la forma más apropiada de evaluar la capacidad competitiva de Colombia, cuyo mercado es el interno. Estos indicadores tampoco permiten ponderar qué factores inciden para lograr el posicionamiento en el mercado internacional que reflejan los ICR en general.

Indicador de Cuasi-Rentas. El indicador de cuasirentas, se utilizó para evaluar la competitividad de los productores de arroz paddy verde en Colombia, bajo los sistemas de producción secano (SS) y riego (SR), y de los productores de arroz paddy seco en EUA, quienes cuentan con la tecnología suficiente 
para agregar valor en la poscosecha. En la Figura 1 , se presentan las cuasi-rentas para la producción de arroz colombiano bajo SR y SS y para USA con y sin subsidios, en la que se puede observar la superioridad competitiva de EUA frente a Colombia. En ambos sistemas, las cuasi-rentas del arroz colombiano lograron en promedio, valores positivos en el periodo analizado, 40,92 USD/ha en SR y 52,74 USD/ha en SS, no obstante, en algunos años se dieron valores negativos; EUA logró en promedio cuasirentas de 348,31 USD/ ha (sin subsidios), con valores negativos entre 1999 y 2002 . Valores positivos de éste indicador, representan ventajas competitivas de un sector en el producto analizado, pero cuando se usa de forma comparativa, el país con el mayor valor de cuasirentas es más competitivo que los demás. Mientras que, valores negativos equivalen a situaciones no competitivas, implicando que los ingresos no son lo suficientemente altos como para cubrir los costos variables (Linares y Gutiérrez, 2002). A continuación se desagrega el indicador de cuasirentas y los resultados obtenidos en cada uno de sus componentes, para comprender los valores anteriormente expuestos.
En general, el margen de contribución en ambos sistemas productivos de arroz en Colombia es muy bajo porque el precio al productor apenas cubre los costos de producción, en algunos años llega a ser menor (datos no mostrados). En promedio, el SS obtiene mayor valor de cuasi rentas con respecto al SR, debido a que SS no incurre en costos de irrigación, los cuales llegan a representar hasta el $11 \%$ de los costos totales en el SR. No obstante, el valor del arriendo de la tierra, que puede llegar a ser hasta del $21 \%$ de los costos en las zonas con riego, es el factor de mayor peso en los costos totales; el costo del arriendo fue, para el SR más de cinco veces el valor de arriendo del SS. En 2010, se pagaron en promedio por semestre, en tierras que contaban con infraestructura de riego \$1.758.886 por hectárea y en aquellas bajo sistema secano \$384.311 por hectárea (FEDEARROZ, 2013).

En el período de análisis, los rendimientos evidenciaron una diferencia importante entre los sistemas productivos colombianos, ya que SR alcanzó valores medios de 5,3t/ha por semestre, frente a 4,0t/ha en SS. Así, los mayores retos en materia de eficiencia para el SR, están

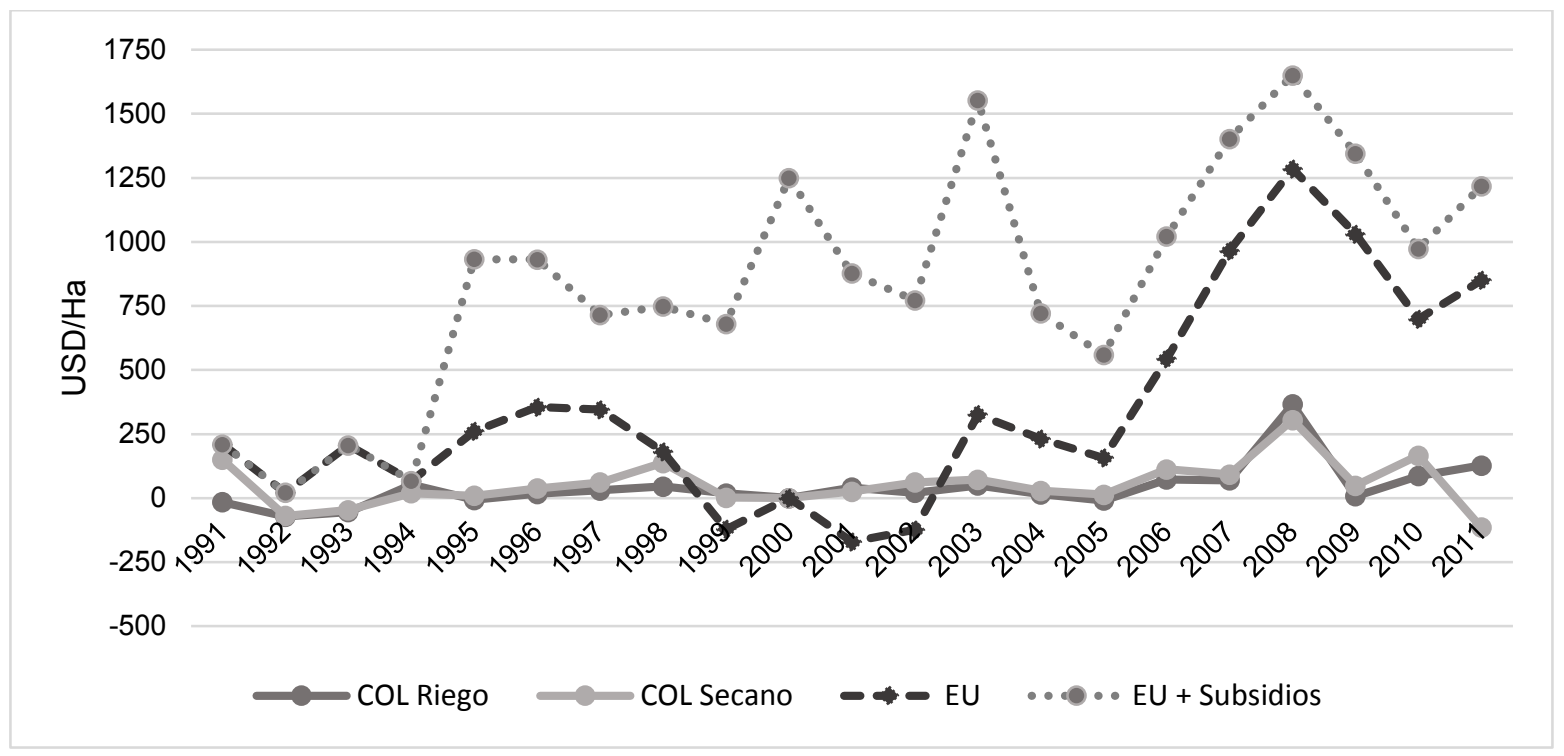

Figura 1. Cuasi-rentas Colombia sistema con riego y secano Vs Estados Unidos (pesos constantes año 2000). 
relacionados con los costos de producción, especialmente con el costo de arriendo, por lo que se debería mejorar sus rendimientos, a fin de contrarrestar el efecto de los costos de producción. En la zona centro, los agricultores manifiestan que los dueños de la tierra suelen aumentar el precio del arriendo cuando en el semestre anterior se alcanzó un buen precio del paddy verde, de tal forma que parte de la ganancia del agricultor pasa directamente al bolsillo del terrateniente. Los productores colombianos en SR que pagan arriendo, son los más vulnerables a los choques en un escenario de fuerte competencia como el que se genera con el TLC.

Aunque el rendimiento es mayor en SR, este indicador sigue siendo menor, comparado con EUA, donde los rendimientos en promedio fueron 7,1t/ha es decir, un 33\% más que en el SR y $77,5 \%$ más que en SS. En consecuencia, en Colombia es necesario un incremento de la productividad y una reducción de los costos de producción para lograr en un plazo mediano, mayores índices de competitividad frente al mercado internacional (FEDEARR0Z, 2012a). Por su parte, los costos de producción en Colombia, son mayores en un $15,3 \%$ en el SR y $11 \%$ en el SS con respecto a EUA, donde, los costos de producción fueron de 114,5 USD/t entre 1991 y 2011. Por otro lado, los precios al productor en EUA, fueron en promedio un 15,5\% mayores a los de Colombia. Cabe resaltar que el precio corresponde al arroz paddy seco, por tanto, se logran altos niveles de rentabilidad.

Las cuasi-rentas en EUA, se calcularon a precio de mercado y con subsidios, dado el peso que tienen estos en los ingresos de los productores. Para ambas situaciones, las cuasi rentas fueron mayores a los resultados obtenidos para Colombia. La cuasi renta promedio, para el periodo evaluado, fue de $348,3 U S D /$ ha sin subsidios, esto es, que la producción estadounidense es 8,5 veces más competitiva que SR en Colombia y 6,6 veces más que en SS. En el período de análisis, los subsidios otorgados por el gobierno de EUA a sus arroceros, estuvieron entre 274,5 y 1.249,9USD/ha, de allí que, el indicador de cuasi-rentas, en la segunda evaluación fuera más alto. El promedio de las cuasi-rentas con subsidios, fue de 850USD/ha, más de dos veces que sin ellos. La mayor competitividad de EUA, puede explicarse en parte, por los rendimientos entre 1991 y 2011; el rendimiento promedio en un año de producción en EUA fue 7,1t/ha. En tal periodo, los rendimientos estadounidense más bajos, fueron de 6,2t/ha, valor que no fue alcanzado en ningún semestre, por ninguno de los dos sistemas de producción en Colombia. Tales rendimientos se explican en eficientes prácticas de cultivos basadas en el uso de tecnología avanzada y un constante trabajo por mejorarlas.

Las cuasi rentas, también se evaluaron comparativamente entre EUA y la Zona Centro (ZC) colombiana, la cual presenta los mejores rendimientos a nivel país predominando la producción en SR. La ZC, tiene una gran fortaleza para la producción de arroz dada la mayor oferta tecnológica y las acciones de transferencia de tecnología que se brindan (Espinal et al., 2005). Entre 1996 2011, los rendimientos de ZC fueron 6t/ha semestral, $11 \%$ mayores que el promedio nacional con riego y 46\% más que el promedio nacional secano. En el mismo período, los arroceros de la ZC obtuvieron un margen de ingresos de 151,7 USD/ha, superando en $153 \%$ los ingresos promedio nacional con riego y en un $132 \%$ promedio nacional secano, mientras que el margen de ganancias, fue del 14\% frente al 22\% logrado por los arroceros en EUA. Las cuasi rentas promedio de la ZC (Figura 2), fue 3 veces menor que para EUA sin subsidios y seis veces con subsidios. 


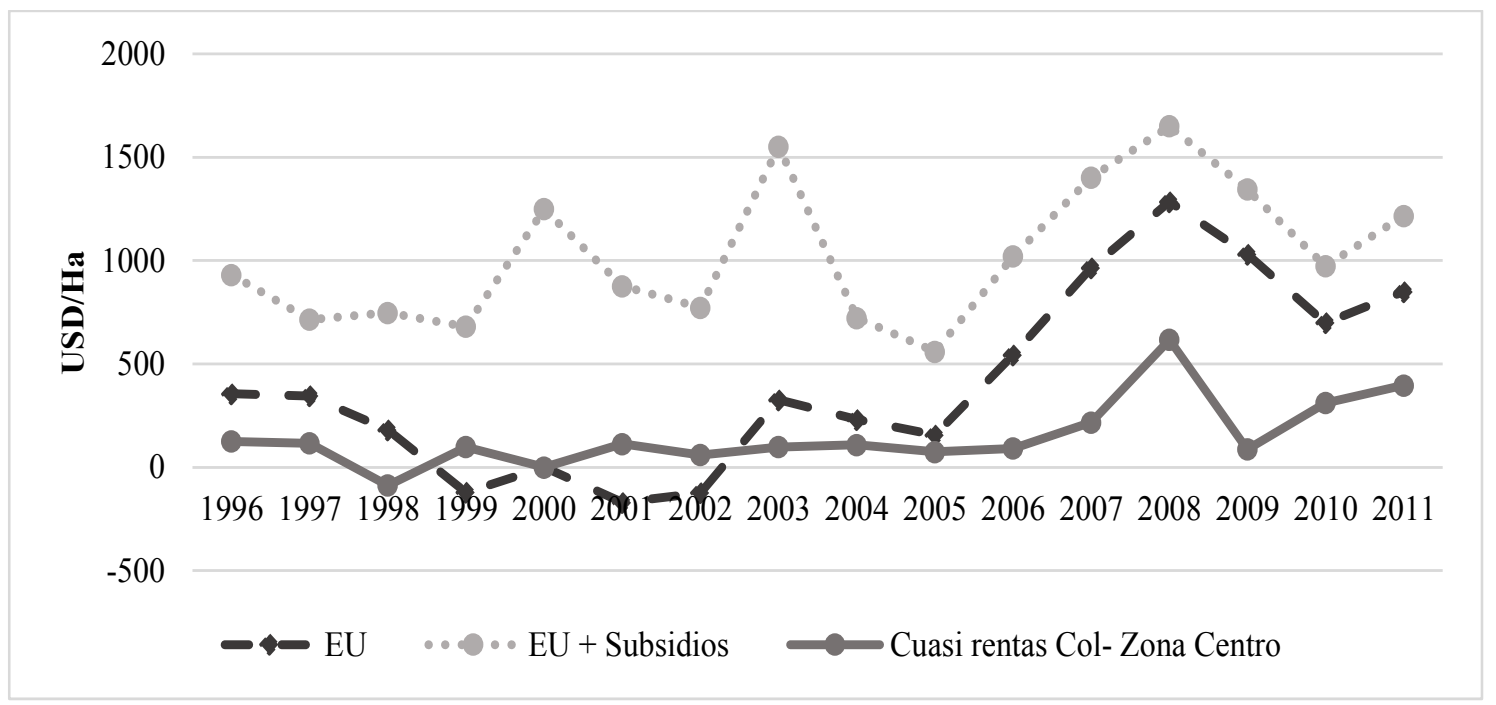

Figura 2. Evaluación de las cuasi rentas de la Zona Centro Colombia y nacional de Estados Unidos (1996 - 2011) (pesos constantes año 2000).

Los rendimientos promedios en el periodo 19962011 de EUA, fueron un 23\% mayores que en la zona más productora de Colombia; por su parte, los costos de producción de la zona centro, fueron un 25\% mayores que los estadounidenses. La diferencia en los rendimientos de ambos países, se puede explicar principalmente en las prácticas de cultivo y el manejo agronómico, en lo que EUA parece ser más eficiente. En EUA, antes de la siembra, se realiza la adecuación del suelo, lo que incluye la nivelación del terreno con el fin de facilitar la inundación y posterior drenaje del agua (Hardke y Scott, 2013). Por su parte, en Colombia, según el más reciente Censo Nacional Arrocero, el $85,7 \%$ de los lotes sembrados en el semestre A y el $81,1 \%$ en el semestre $B$, no realizó ningún tipo de adecuación de suelos (FEDEARROZ, 2008). La preparación adecuada del terreno, garantiza el manejo eficiente de la lámina de riego y el desarrollo adecuado, de las plantas de arroz; no hacerlo se traduce en disminuciones en los rendimientos del cultivo (Mogollón, 2006).

En EUA, toda la producción arrocera se hace bajo riego, contando con infraestructura especializada para dicho fin (Snyder y Slaton, 2001) mientras que en Colombia el 64\% del área arrocera cuenta con sistema de riego, el 31,8\% es secano mecanizado y el $4,2 \%$ es secano convencional (FEDEARROZ, 2008). El riego, constituye un factor fundamental no sólo para el desarrollo de la planta sino en la disminución del uso de insumos; una adecuada infraestructura de riego, es clave para aumentar los niveles de productividad del cultivo.

Las semillas utilizadas para la siembra en EUA, han sido desarrolladas específicamente para cada estado productor por centros de investigación, teniendo en cuenta las condiciones agroecológicas, factor que influye en los altos rendimientos obtenidos (Wilson et al., 2013). Por su parte en Colombia, un poco menos de la mitad de los lotes son sembrados con semillas no certificadas, que corresponden a "semilla de bulto" o parte de lo mejor de la cosecha guardada por los agricultores para la siembra siguiente (FEDEARR0Z, 2008), lo que no siempre garantiza mayores rendimientos. Otro aspecto relevante, es el manejo de la nutrición; los productores estadounidenses hacen fertilizaciones basadas en necesidades nutricionales medidas en campo, en estudios de asimilación de elementos químicos, análisis de suelos constantes y prácticas de recuperación de los mismos, además de actividades que buscan un 
equilibrio entre las dosis requeridas y las dosis aplicadas. De igual manera, el manejo fitosanitario se basa en mediciones del avance del organismo que causa la afección, sin embargo, estrictas prácticas preventivas son efectuadas para garantizar un control efectivo en el momento que sea requerido (Moldenhauer y Slaton, 2003). En Colombia, a pesar que existen algunas investigaciones por parte de entidades como FEDEARROZ, sobre los requerimientos nutricionales de las variedades utilizadas, es común el uso de tres principales fertilizantes independientemente de la zona y de la variedad: urea $\mathrm{CO}\left(\mathrm{NH}_{2}\right)_{2}$ fosfato diamónico DAP, cloruro de potasio (KCl) (Blanco, 2003), los cuales suelen ser aplicados como una "receta" y no basado en análisis edafológicos ni en necesidades nutricionales de las plantas.

En el análisis de los datos, se logró evidenciar que los costos de producción explican buena parte de la diferencia en los niveles de competitividad, pues el margen de rentabilidad, es muy bajo para Colombia y relativamente altos para EUA; en promedio para el período de análisis (1991 - 2011), el margen de rentabilidad de los productores de EUA y colombianos en SR y SS fueron 21, 3,4 y 8,2\%, respectivamente. En ese sentido, los productores de EUA han desarrollado economías de escala aumentando el tamaño de la finca (Childs, 2001); en los últimos años, se ha dado una reducción significativa del número de granjas, pasando de 11.212 en 1992 a 6.084 en 2007 y simultáneamente, con un aumento del área promedio pasando de $100 \mathrm{a}$ 200 hectáreas para el mismo periodo de tiempo (Baldwin et al., 2011). La captura de economías de escala, permite que los productores sean más eficientes en el uso de los recursos a través de una mayor especialización y un menor costo unitario. A pesar que los insumos (agroquímicos) tienen una gran participación dentro de la estructura de costos de ambos países, alrededor de un 45\% para Colombia y $35 \%$ para EUA, los productores estadounidenses tienen a favor el hecho que EUA es productor de insumos, mientras que Colombia es importador neto. El gasto en insumos representó para el año 2011 alrededor de $\$ 2.000 .000$ por hectárea por semestre para Colombia, comparado con aproximadamente $\$ 800.000$ hectárea por año en EUA. Los fertilizantes representaron 23\% en SR y $11 \%$ en SS de los costos de producción en el 2008, lo cual se explica no sólo por las abundantes cantidades implementadas sino porque la fertilización en muchas ocasiones se hace de manera empírica o por calendario (Espinal et al., 2005), mientras que el uso de la tecnología y mecanización en el cultivo del arroz en EUA, tienen la mayor participación dentro de la estructura de costos, así como el arriendo de la tierra. Según Childs (2001) esto se debe principalmente a la competencia que existe entre el uso de la tierra para la agricultura o para procesos de urbanización.

El precio, es un factor determinante de competitividad, pues disuade al consumidor en su decisión de compra. Igualmente, se utiliza como argumento para elegir la importación de bienes al consumo del producto nacional y por lo tanto, para la firma de acuerdos comerciales entre países (Díaz, 2012). En el caso de los productos agrícolas, el precio de los commodities, es el reflejo de sus costos de producción, los subsidios a la producción y los términos de la negociación comercial. En el caso de los productores estadounidenses, estos comercian arroz paddy seco, un producto con un mayor valor agregado como efecto de la infraestructura para almacenamiento y secado con que cuentan los productores, mientras en Colombia el secado es efectuado por los molinos, por lo tanto, no influye en el precio al productor de la materia prima.

En el caso de Colombia, un factor que afecta negativamente los precios al productor, es el contrabando de arroz a un menor precio que entra por la frontera terrestre con Venezuela y Ecuador, lo que deprime el precio interno; los esfuerzos del gobierno colombiano, son pocos en éste sentido (FEDEARROZ, 2012b).; sin embargo, desde finales de 2014 y el primer semestre de 2016 el contrabando se ha visto contrarrestado por la devaluación de la moneda colombiana, con lo que la producción nacional se hizo más competitiva. 
Dado que los subsidios hacen parte fundamental de la estructura de ingresos de la producción arrocera en EUA, se hizo una revisión de éstos. El sector agropecuario estadounidense, es considerado un asunto clave para la seguridad nacional del país y su soberanía alimentaria (Díaz, 2012) por lo cual, sus políticas agrícolas están enmarcadas en el papel fundamental que tiene la agricultura y en la influencia de dicho país en los mercados internacionales de los bienes agropecuarios (Garay et al., 2005). Dichas políticas se encuentran estipuladas en las leyes agrícolas sancionadas cada cinco años; la actual ley agrícola "The Food, Conservation, and Energy Act of 2008", estipula los subsidios otorgados al sector arrocero estadounidense durante el período de análisis de este estudio; según el USDA (s.f.) incluyen: préstamos para la comercialización, pagos directos (DPs por sus siglas en inglés) y pagos contra cíclicos (CCPs por sus siglas en inglés), además de subsidios para la asistencia permanente de desastres; esta ley expiró en 2012 y fue reemplazada por una nueva reforma a la ley agrícola denominada "Farm Act 2014", que deroga los programas anteriormente mencionados y crea los programas cubrimientos a la pérdida en precios y el cubrimiento al riesgo agrícola (PLC y ARC, respectivamente, por sus siglas en inglés) (Hungerford, 2016).

Diversos autores Tejada (2009), Griswold (2007) y Basco et al. (2003) describen estos subsidios y su efecto en la conducta económica del productor arrocero de EUA, quiénes básicamente no dejan de producir aunque el precio no sea costo efectivo, pues los subsidios compensan ampliamente la situación, tal como se aprecia en la figura 1, cuando las cuasi rentas de EUA sin subsidios, es negativa para unos años, pero los subsidios son más elevados en el mismo período, generando altas cuasi rentas. Suárez (2007) plantea que los subsidios agrícolas en EUA pueden configurar coyunturalmente casos de dumping, lo que afecta el desarrollo agrícola de los países que compiten con productores de productos básicos de EUA, ya que en su propio mercado local, los precios de EUA son más bajos.
Para el caso del arroz estadounidense, el IATP (2003) demostró la configuración de dumping, estableciendo que los costos de producción superaban en un 26\% los precios de exportación. Por su parte, en la revisión de la política agrícola Colombia como la ley general de desarrollo agropecuario (Ley 101 de 1993) (Congreso de la República, 1993) y la ley que crea los fondos de fomentos agrícolas (ley 67 de 1983) (Congreso de la República, 1983), no se logró identificar subsidios directos y permanentes a los productores de arroz diferentes de aquellos que recibe cualquier productor agropecuario del país, cómo los créditos agrícolas con bajas tasas y los subsidios para cubrir parte del costo de la asistencia técnica, entre otros. Para los arroceros colombianos la falta de subsidios y la competencia en el marco del TLC con Estados Unidos y su política de subsidios agrícolas, constituye una clara amenaza a su permanencia en el mercado.

Por otro lado, la deficiente infraestructura económica del país y del sector agrícola, contribuye igualmente a la baja competitividad de la producción arrocera colombiana. Nos referimos a la limitada y debilitada malla vial, a los altos costos de los peajes y del combustible, lo que ha sido una crítica permanente al modelo de apertura económica desde su establecimiento en los noventa. En el subsector arrocero las falencias se encuentran en el bajo número de distritos de riego a nivel nacional, la inexistente infraestructura para el secado y almacenamiento en manos de los agricultores, la escasa transferencia de tecnología y los pobres recursos para la investigación en el sector. En estas condiciones es difícil contemplar un aumento en la productividad.

También se ha identificado que la asociatividad es un factor determinante en la capacidad competitiva de EUA, donde son usuales las alianzas estratégicas $\mathrm{y}$ asociaciones de productores, con el fin de aumentar la eficiencia del sistema productivo, mediante el uso de los avances en la tecnología agrícola y del proceso de comercialización. En 
Colombia no existe la cultura de la asociación para la producción agrícola y el arroz no es la excepción. Por todo lo anteriormente mencionado, la seguridad alimentaria medida por la disponibilidad de alimentos, acceso a los alimentos y estabilidad según la FAO (2006), en el marco del TLC podría estar en riesgo, pues el producto estadounidense, entrará al país a un menor precio, desestimulando la producción interna y el área sembrada como se evidencia en el estudio realizado por Ramírez y Martínez (2005) por lo tanto aumentando la dependencia por las importaciones.

Así, en un escenario de alta dependencia y altos precios internacionales del arroz, los colombianos en niveles de pobreza aproximadamente el $30,6 \%$ de la población total, tendrían los mayores problemas de acceso a éste alimento básico. Ramírez et al. (2014), estimaron que un aumento del $20 \%$ en el precio del arroz, implica que la tasa de indigencia aumenta de 10,4 a 10,8\% y la tasa de pobreza aumenta de 32,7 a $33,6 \%$ a nivel nacional. El efecto es especialmente marcado en zonas rurales, donde la incidencia de la indigencia aumenta de 22,7 a 23,5\% y la incidencia de la pobreza aumenta de 46,9 a $47,9 \%$.

Sin duda, los pequeños productores arroceros del país, serán los primeros en ver su seguridad alimentaria amenazada, al ser desplazados por las importaciones. Según Garay et al. (2010) con la entrada en vigencia del TLC, se estima que los precios internos al productor arrocero serán inferiores en un $18 \%$ con respecto al periodo 1998-2002, lo que implica una diminución del ingreso bruto aproximado del 32\%. Garay et al. (2005) también estiman la pérdida del $17 \%$ del empleo que genera el sector, en un escenario de cero aranceles para el arroz importado desde EUA. Las ventajas competitivas de los productores de arroz estadounidense, son innegables y están fundamentadas en la tecnología, en tanto que los cuantiosos subsidios directos a la producción, también hacen un aporte significativo para lograr un precio muy competitivo para el comercio internacional.
Todo el proceso productivo, debe mejorarse en cuanto a la adecuación del suelo, el manejo del agua, que se utiliza en exceso, uso de semilla certificada, fertilizaciones mínimas requeridas, manejo integrado de plagas; igualmente, los productores deben tomar mayor participación en la cadena de valor y para ello se requiere apoyo del gobierno nacional, que a través de una política sectorial, financie y apoye infraestructura de secado y almacenamiento, de tal forma que el producto final del productor agrícola sea el arroz paddy seco.

\section{CONCLUSIONES}

Los indicadores de competitividad revelada y cuasi rentas evaluados en este estudio, para Estados Unidos, demuestran su alta competitividad como productor y exportador de arroz, lo que en el marco del TLC con Colombia, implica la oportunidad de que afiance su presencia en el mercado de arroz colombiano. En tanto que los resultados de los indicadores de competitividad para la producción de arroz en Colombia, demuestran su no participación en el mercado mundial de arroz y su frágil posición en el mercado nacional. Por lo anterior, los agricultores arroceros colombianos deberán, en el período de protección o gracia, establecido por el TLC, mejorar su competitividad con el fin de sostener su posición en el mercado interno. Los resultados también indican, que el esfuerzo del sector arrocero colombiano deberá estar enfocados a mejorar eficiencia económica, vía reducción de costos y eficiencia técnica, con el aumento de rendimientos.

\section{REFERENCIAS BIBLIOGRÁFICAS}

AGRONET. 2014. Área, producción y rendimiento nacional por cultivo. En: http://www.agronet.gov.co/ estadistica/Paginas/default.aspx.; consulta: agosto, 2014.

ALVARADO, Y.; LEYVA, M.; MUÑOZ, I.; SÁNCHEZ, 0. 2009. Oferta, demanda, balanza comercial y competitividad del pescado de México en el mundo, Economic analysis working papers, No. 2009,8. Coruña, España. 
BALDWIN, K.; CHILDS, N.; DOHLMAN, E.; FOREMAN, L. 2011. Consolidation and structural change in the U.S. rice sector. USDA - Economic Research Service (RCS11D-01). United States Department of Agriculture. Washington. Estados Unidos. 28 p.

BALZARAVICIENE, S.; PILINKIENE, V. 2012. Comparison and review of competitiveness indexes: towards the EU policy. Rev. Economics and management. 17(1):103 - 109.

BASCO, C.; BUCCELLATO, I.; DELICH, V.; TUSSIE, D. 2003. La nueva ley de seguridad agrícola y de inversión rural de los Estados Unidos (Farm Bill): Un análisis de sus implicancias comerciales. Serie Comercio Internacional. CEPAL. Santiago de Chile. 34 pp.

BLANCO, J. 2003. Manejo integral de suelos con énfasis en el cultivo del arroz. Proyecto PRONATTA. En: www. agronet.gov.co/www/docs_si2/Manejo $\% 20 \mathrm{del} \% 20$ suelos\%20para\%arroz.pdf. Consulta: julio, 2014.

CHILDS, N. 2001. Rice: background and issues for farm legislation. US Department of Agriculture. Economic Research Service USDA. 14 p.

CONGRESO DE LA REPÚBLICA (1993). Ley 101 de 1993, Ley general de desarrollo agropecuario y pesquero. En: https://www.minagricultura.gov.co/Normatividad/Leyes/Ley\%20101\%20de\%201993.pdf; consulta: septiembre, 2016.

CONGRESO DE LA REPÚBLICA (1983). Ley 67 de 1983, Por la cual se modifican unas cuotas de fomento, y se crean unos fondos y se dictan normas para su recaudo y su administración. En: http://www.fedearroz.com.co/ normas/LEY\%2067\%20DE\%201983.pdf; consulta: septiembre, 2016.

DNP. DEPARTAMENTO NACIONAL DE PLANEACIÓN. 2014. Reporte global de competitividad 2014-2015: Foro Mundial, síntesis de resultados para Colombia. En: https://colaboracion.dnp.gov.co/CDT/Desarrollo\%20 Empresarial/Documento_FEM_2014.pdf.; consulta: marzo, 2016.

DÍAZ, VG. 2012. Impacto del TLC con Estados Unidos en el sector agrícola colombiano, más riesgos que oportunidades (caso arroz). Rev. CIFE. 14(20): 8.
ESPINAL, F.; MARTÍNEZ, H.; ACEVEDO, X. 2005. La cadena del arroz en Colombia. Una mirada global de su estructura dinámica 1991-2005. En: MADR: https://sioc. minagricultura.gov.co/Arroz/Documentos/004\%20 - $\% 20$ Documentos $\% 20$ Competitividad $\% 20$ Cadena/004\%20-\%20D.C. $\% 20-\% 20$ Caracterizacion $\% 20$ Arroz.pdf; consulta: diciembre, 2015.

EWG. ENVIOMENTAL WORKING GROUP 2015. Farm subsidy database. En:_https://farm.ewg.org/progdetail. php?fips $=00000 \&$ progcode=rice\&regionname $=$ theUni tedStates; consulta; diciembre, 2015.

FAO. FOOD AGRICULTURE ORGANIZATION. 2006. Informe de políticas: Seguridad alimentaria. En: ftp://ftp.fao.org/es/ESA/policybriefs/pb_02_es.pdf.; consulta: Agosto, 2014.

FAOSTAT. FOOD AND AGRICULTURE ORGANIZATION OF THE UNITED NATIONS STATISTICS DIVISION. 2015. Cultivos y productos de ganadería. En: http://www.fao. org/faostat/es/\#data/TP; consulta: Diciembre, 2015.

FAOSTAT. FOOD AND AGRICULTURE ORGANIZATION OF THE UNITED NATIONS STATISTICS DIVISION. 2012. Food balance and food supply. En: http://faostat.fao. org/site/291/default.aspx; consulta: Diciembre, 2015.

FEDEARROZ. FEDERACIÓN NACIONAL DE ARROCEROS. 2008. III Censo Nacional Arrocero. Bogotá, Colombia. $196 \mathrm{pp}$.

FEDEARROZ. FEDERACIÓN NACIONAL DE ARROCEROS. 2012a. ¿Qué tanta es la amenaza de las importaciones?. En: http://www.fedearroz.com.co/revistanew/arroz500.pdf; consulta: noviembre, 2015.

FEDEARROZ. Federación Nacional de Arroceros. 2012b. Contrabando de arroz. En: http://www.fedearroz.com. co/revistanew/arroz498.pdf; consulta: noviembre, 2015.

FEDEARROZ. Federación Nacional de Arroceros. 2013. Concepto de competitividad. En: http://www.fedearroz.com.co/revistanew/arroz507.pdf; consulta: noviembre, 2015.

FEDEARROZ. FEDERACIÓN NACIONAL DE ARROCEROS. 2014. Discurso instalación del XXXIV congreso nacional arrocero del doctor Rafael Hernández En: http://www.fedearroz.com.co/revistanew/arroz508. pdf; consulta: noviembre, 2015. 
GARAY, LJ. 1998. Colombia: estructura industrial e internacionalización 1967 - 1996. Biblioteca virtual del Banco de la República. Colombia. 630pp. En: http:// www.banrepcultural.org/sites/default/files/libro.pdf.; consulta: agosto, 2016.

GARAY, L.J.; BARBERI, F.; CASTRO, Y.; PERRY, R.; CARDONA, I. 2005. Una visión integral de la agricultura en el TLC con Estados Unidos. En Garay, LJ. La agricultura colombiana frente al tratado de libre comercio con Estados Unidos. Ministerio de Agricultura y Desarrollo Rural. Bogotá D.C. 30 - 40 p.

GARAY, L.J.; BARBERI, F.; CARDONA, I. 2010. Los impactos del TLC desde una perspectiva de estática comparativa. En Garay, LJ. 2010 Impactos del TLC con Estados Unidos sobre la economía campesina en Colombia. Editorial ILSA (Instituto Latinoamericano de Servicios Legales). Santiago de Chile. 45 - 75 p.

GRISWOLD, D. 2007. El costo oculto de los subsidios arroceros de Estados Unidos. Cato Institute. Washington, DC. 11 pp. En: http://www.elcato.org/elcosto-oculto-de-los-subsidios-arroceros-de-estadosunidos; consulta: junio, 2016.

GUTIÉRREZ, A. 1999. Reformas económicas y mejoramiento de la competitividad: El caso de la producción de papa en el Estado Mérida. Rev: Agroalimentaria. 9 (43 - 54): 25.

HARDKE, J.; SCOTT, B. 2013. Water seeded rice, pp. 41-44. En: Hardke, J. (ed.). Rice production handbook. University Of Arkansas Cooperative Extension Service. United States of America. 206 p.

HERTFORD, R.; ESPINAL C. 1996. Desempeño de la agricultura durante el ajuste económico y la apertura de los Países Andinos y en Colombia: implicaciones para la competitividad. Cap. VIII. En: Rojas P, Chavarría H, Sepúlveda S. 1996. La competitividad de la agricultura. IICA. Santa Fe de Bogotá, Colombia. 232 - 235 p.

HUNGERFORD, A. 2016. Agricultural Act Of 2014: Highlights And Implications: Crop Commodity Programs. En: https://www.ers.usda.gov/agriculturalact-of-2014-highlights-and-implications/cropcommodity-programs/; consulta: septiembre, 2016
IATP. INSTITUTE FOR AGRICULTURA AND TRADE POLICY. 2003. United States dumping on world agricultural markets. World Trade Organization (WTO). Minnesota, Estados Unidos. 28 p.

LINARES, Y.; GUTIÉRREZ, A. 2002. La competitividad de la producción de papa (Solanum tuberosum) en el municipio Pueblo Llano, estado Mérida, Venezuela. Agroalimentaria. 7(15):37 - 47.

LOTERO, C.J.; MORENO, M.A.; VALENCIA, A.M. 2005. La competitividad: aproximación conceptual desde la teoría del crecimiento y la geografía económica. Centro de investigaciones económicas. Universidad de Antioquía, Medellín. 28 p.

LOTERO, C.J.; HÉCTOR, P.; DANIEL, V. 2009. La competitividad de los departamentos colombianos desde la perspectiva de la geografía económica. Centro de investigaciones económicas. Universidad de Antioquía, Medellín. 28 p.

MOGOLLÓN, V.J. 2006. Una alternativa para disminuir los costos de producción de los cultivos de arroz en el municipio del Espinal, Tolima. Universidad de la Salle, Bogotá D.C. 111 p.

MOHANTY, S. 2013. Trends in global rice consumption. En: Rice Today, http://irri.org/rice-today/trends-inglobal-rice-consumption; consulta: septiembre, 2016.

MOLDENHAUER, K.; SLATON N. 2003. Rice Growth and Development. pp. 7-14. En: Smith, W.; Dilday, R. Rice, origen, history, technology, and production. Jhon Wiley and Sons., Inc. United States of America. 643 p.

PERFETTI, J.J.; BALCÁZAR, A.; HERNÁNDEZ, A.; LEIBOVICH, J. 2013. Políticas para el desarrollo de la agricultura en Colombia. SAC y FEDESARROLLO. Bogotá. 248 p.

RAMÍREZ, M.; MARTÍNEZ, H. 2005. Transmisión de precios y efectos en producción y consumo. pp. 403 - 494. En: Garay, L; Barberi, F; Espinosa, A (editor). 2005. La agricultura colombiana frente al tratado de libre comercio con Estados Unidos. Ministerio de Agricultura y Desarrollo Rural de Colombia. Bogotá. 556 p. 
RAMÍREZ, JM.; GÓMEZ, D.; BECERRA A. 2014. Efectos sobre bienestar y pobreza de la política comercial agrícola: el caso del arroz en Colombia. En: FEDESARROLLO, http://www.fedesarrollo.org.co/wp-content/ uploads/2014/03/working-paper-No-63.pdf; consulta: septiembre, 2015.

ROJAS, P.; SEPÚLVEDA, S. 1999. ¿Qué es la competitividad?, pp. 26 - 28. En: Rojas, P.; Chavarria, H.; Sepúlveda, S. Competitividad de la agricultura: cadenas agroalimentarias y el impacto del factor localización espacial. IICA, San José, Costa Rica. 379 p.

SCHWARTZ, M.; IBARRA, K.; ADMA, C. 2007. Indicadores de competitividad de la industria exportadora chilena de palta (aguacate). pp 1 - 10. En: Proceedings VI World Avocado Congress. Viña Del Mar, Chile.

SUÁREZ, A. 2007. El modelo agrícola colombiano y los alimentos en la globalización. Ediciones Aurora. Bogotá. 221 p.

SNYDER, C.; SLATON, N. 2001. Rice Production in the United States - An Overview. Rev. Better Crops. 85(3):3 - 7 .

TEJADA, A. 2009. La nueva ley agrícola estadounidense (Farm Bill 2008 - 2013) y sus repercusiones económicas y comerciales. En: http://www.inai. org.ar/archivos/notas/La\%20nueva $\% 20$ Ley $\% 20$ Agr\%C3\%ADcola\%20de\%20Estados\%20Unidos.pdf; consulta: enero, 2016.

USDA. (s.f.) UNITED STATES DEPARTMENT OF AGRICULTURE. Natural Resources Conservation Service. 2008 Farm Bill Archive. En: https://www.nrcs.usda. gov/wps/portal/nrcs/detail/national/programs/ farmbill/archived/?cid=stelprdb1245079; consulta: Diciembre, 2015.

WILSON, C; MOLDENHAUER, K.; CARTWRIGHT, K; HARDKE, J. 2013. Rice cultivars and seed production, pp. 21-30. En: Hardke, J. (ed.). Rice production handbook. University Of Arkansas Cooperative Extension Service. United States of America. 206 p. 